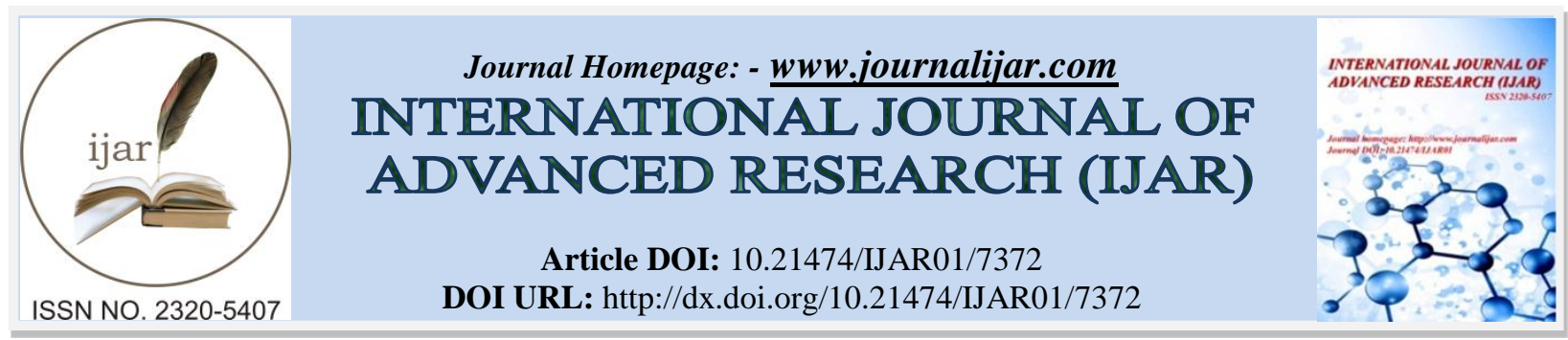

RESEARCH ARTICLE

\title{
A CLINICAL STUDY OF MICROALBUMINURIA IN NON-DIABETIC CORONARY ARTERY DISEASE PATIENTS IN AN INDUSTRIAL HOSPITAL.
}

1. Medical Officer Health Department Kashmir.

2. Specialist Urology, Al Raffah Hospital, Oman.

\section{Manuscript Info}

Manuscript History

Received: 08 May 2018

Final Accepted: 10 June 2018

Published: July 2018

\section{Abstract}

Objective: The objective of our study was to study the prevalence of microalbuminuria in non-diabetic patients with Ischemic heart disease and to identify whether it can be used as an independent marker of Ischemic heart disease or as a screening test for ischemic heart disease in non- diabetic patients.

Material and methods: This was a hospital based prospective study. All patients presenting with coronary artery disease to our hospital from May 2014 to Sep 2016 were be included. Total number of patients included in our study were 50 as most patients with IHD were diabetic as well and were excluded from the study. All these patients were subjected to detailed history and physical examination. All baseline investigations, ECG, Chest X-ray, lipid profile and urine for microalbumin by Turboux method were done on these patients.

Results: - Total 50 patients were included in the study (39 males and 11 females). The mean age of the patients was $58.22 \pm 10.15$ years. Out of 50 patients, $30(60 \%)$ had microalbuminuria. There were 24 males $(61.5 \%)$ and 6 females $(54.5 \%)$ with abnormal microalbuminuria levels. Most of the patients with microalbuminuria were in age range of 45-65years. There was statistically significant association of microalbuminuria with hypertension, LDL >100mg/dl and HDL<35 $\mathrm{mg} / \mathrm{dl}$ whereas no association was seen between microalbuminuria and other risk factors of IHD.

Conclusion: From our study we concluded that there is high prevalence of microalbuminuria in non-diabetic ischemic heart disease patients but the association between two is not statistically significant. At present microalbuminuria cannot be used as an independent marker of IHD nor can it be used as a screening tool for IHD in general population unless further prospective studies with large sample size and comparative analysis against normal controls are done in future.

Copy Right, IJAR, 2018,. All rights reserved.

\section{Introduction:-}

Ischemic Heart Disease has an estimated prevalence of 6-9\% in the general population. The mortality rate of acute myocardial infarction is approximately 30\%. 1 in 25 patients who survives the initial hospitalization die in the first year after acute myocardial infarction (1). In India, coronary artery disease is equally important and its incidence has

Corresponding Author:- Nuzhat Tabasum.

Address:- Medical Officer Health Department Kashmir. 
increased in the last 2 decades. Many prospective clinical studies have identified a series of independent risk factors for myocardial infarction, stroke and peripheral vascular disease, among which the pre-existence of atherosclerotic vascular disease, age, male gender, a positive family history of premature atherosclerotic disease, smoking, diabetes mellitus, hypertension, hypercholesterolemia, hypertriglyceridemia and low HDL cholesterol are considered as classical risk factors. However, there are some emerging risk factors which can be used independently. Currently there is an intense discussion whether they should be introduced into routine risk assessment. This especially concerns Lipoprotein (a), CRP, fibrinogen, homocysteine and microalbuminuria.

A consensus conference in 1995 defined microalbuminuria (MA) in individuals with diabetes as an abnormal urinary excretion rate of albumin between the range of $20-200 \mu \mathrm{g} / \mathrm{min}$ or $30-299 \mathrm{mg} / \mathrm{day}$. In this disease, microalbuminuria is an early predictor of renal damage. It takes usually more than 5 years for type I diabetics to have microalbuminuria. Microalbuminuria has also been implicated as a sensitive indicator of non-renal disease (2, 3). In fact microalbuminuria occurs in response to acute inflammatory conditions such as ischemia (4) , trauma and thermal injury (5), surgery (6), pancreatitis (7) and inflammatory bowel disease (8). In all these conditions the degree of microalbuminuria is proportional to the severity of the inflammatory insult, predictive of outcome and not associated with any other feature of renal impairment (9).

Amongst all these, the association of microalbuminuria with cardiovascular diseases is very important owing to the burden of cardiovascular morbidity and mortality The Prevention of Renal and Vascular End Stage Disease (PREVEND) study concluded that urinary albumin measurement may be useful in early risk profiling and prevention of cardiovascular disease as it is independently associated with increased cardiovascular risk factors and cardiovascular morbidity. Myocardial infarction is the most important manifestation of cardiovascular diseases. Microalbuminuria is an early response to myocardial infarction .It is also proportional to the infarct size $(10,11)$. A study by Berton et. al., showed that microalbuminuria occurs in acute myocardial infarction and predicts early mortality $(12,13)$. In a cross sectional study in western India in 2002 , microalbuminuria was shown to be associated with carotid intima - media thickening and coronary artery disease (13).

It has been hypothesized that microalbuminuria is an indicator of wide spread endothelial cell dysfunction leading to increased penetration of atherogenic protein in the arterial wall. In the 1st MONICA (Monitoring Trends and Determinants of Cardiovascular Diseases) study in Copenhagen County, individuals with persistent microalbuminuria had increased transvascular albumin leakage to a level similar to that seen among individuals with severe clinical atherosclerosis.(13)Though the underlying mechanism remains undetermined, it has been hypothesized that the magnitude of albumin excretion in urine reflects the degree of atherosclerosis. If this is so, the urinary albumin excretion should be high in patients with IHD even in non diabetics. Few studies have indicated that microalbuminuria is common in nondiabetic, nonhypertensive population and is considered to be an independent indicator of cardiovascular risk factors and cardiovascular mortality. $(14,15)$ In clinical practice, a spot-urine sample is collected when the patient visits either the general practitioner or the health care office, where the screening takes place.

\section{Aim of the study:-}

1. To study the prevalence of microalbuminuria in non-diabetic patients with Ischemic heart disease.

2. To identify the association of microalbuminuria and other known risk factors of Ischemic heart disease.

3. To identify whether microalbuminuria can be used as an independent marker of Ischemic heart disease.

4. To identify whether microalbuminuria can be used as a screening test for ischemic heart disease in non- diabetic patients.

\section{Material and methods:-}

This was a hospital based prospective study conducted in a tertiary care referral hospital of Northern Railways at New Delhi which caters the need of Railway beneficiaries from North India constituting about 1.5 million people. All patients presenting with coronary artery disease to our hospital from May 2014 to Sep 2016 were be included in our study. Total number of patients included in our study were 50 as most patients with IHD were diabetic as well and were excluded from the study. All these patients were subjected to detailed history and physical examination. All baseline investigations, ECG, Chest X-ray, lipid profile and urine for microalbumin by Turboux method were done on these patients. 


\author{
Inclusion Criteria:- \\ The study will include 50 cases who present with history of: \\ Angina on Exertion \\ Unstable Angina \\ Acute Myocardial Infarction \\ Post MI patients (post-CABG, post-PTCA, Medically treated patients) \\ Exclusion Criteria:- \\ 1. Known diabetics \\ 2. CKD patients \\ 3. Systemic infection \\ 4. Urinary tract infection \\ 5. Inflammatory conditions like rheumatoid arthritis
}

This method is based on the Principle of Liquid phase immunoprecipitation assay with nephelometric end-point detection. In this, Antiserum to albumin is diluted in buffer \& added to urine sample \& light scattering caused by $\mathrm{Ag}-\mathrm{Ab}$ complexes is measured after incubation, which is directly proportional to albumin concentration. Precalibration of curve is defined by batch specific parameters on magnetic card. The single point calibration to check the curve during assay is made with calibrator solution. The results are expressed as conc.mg/L. The method is fast \& sensitive to very low conc. of albumin. The reagent used in this method contains azide (swine).Urine albumin control is available in kit.

Sample Collection:-Spot urine sample for screening

\title{
Results:-
}

It was a hospital based prospective study. Total 50 patients were included in the study (39 males and 11 females). The mean age of the patients was $58.22 \pm 10.15$ years. Family history of IHD was present in $30 \%$ patients, while smoking history was present in $66 \%$ of patients (only one female patient had smoking history compared to $82 \%$ males). Hypertension was present in $78 \%$ of patients of which females had a higher percentage (Female to Male $81.8 \%$ to $76.9 \%$ ). $40 \%$ patients had history of previous cardiac event. $21(42 \%)$ patients had raised level of cardiac enzymes on admission. Out of 50 patients, $30(60 \%)$ had microalbuminuria in our study. There were 24 males $(61.5 \%)$ and 6 females $(54.5 \%)$ with abnormal microalbuminuria levels. Most of the patients with microalbuminuria were in age range of 45-65years. (TABLE 1) There was statistically significant association of microalbuminuria with hypertension, LDL $>100 \mathrm{mg} / \mathrm{dl}$ and $\mathrm{HDL}<35 \mathrm{mg} / \mathrm{dl}$ whereas no association was seen between microalbuminuria and other risk factors of IHD. (TABLE2)

\section{Discussion:-}

Worldwide, ischemic heart disease is assuming an increasing role as a major cause of morbidity and mortality. IHD will become a major disease burden in India by the year 2020. From 1990 to 2020, the proportion of worldwide deaths due to cardiovascular disease including IHD is expected to increase from $28.4 \%$ to $33 \cdot 7 \%$. In terms of years of life lost, cardiovascular disease will jump from fourth to first place. For premature death and disability, cardiovascular disease will move from fifth to first place. However, despite the rising tide of this tragic disease, the physicians' ability to predict the development of a cardiovascular event is limited by the low prognostic specificity of the traditional risk factors for atherosclerosis. This justifies the ongoing search for new bio markers of atherosclerosis. To target preventive strategies, risk stratification of the population should be effective. There are many reports emanating from the literature about microalbuminuria as an independent risk factor for development of ischemic heart disease. Hitherto, microalbuminuria was considered as a marker of endothelial dysfunction in diabetes mellitus, but many studies have shown microalbuminuria is an effective marker of generalised vascular dysfunction even in non-diabetic population.

This study was done to find out the prevalence of microalbuminuria in non- diabetic patients with IHD and whether it can be used as a marker or as a screening test in these patients. All the cases in the present study had a normal renal function ( $\mathrm{Sr}$ creatinine $\leq 1.5 \mathrm{mg} / \mathrm{dl}$ ). Microalbuminuria, in these patients was therefore not related to renal dysfunction. Our study in this respect agrees with the views of Peter Gosling, who considered it to be a sensitive indicator of nonrenal disease (9). Patients with renal or urinary tract disease or diabetes mellitus were excluded from 
this study, as urinary albumin excretion is elevated in these diseases for reasons other than atherosclerosis. The use of early morning urine sample was preferred to minimize the contribution of posture and exercise to urinary albumin excretion which are known to cause microalbuminuria.

Out of 50 patients in our study, $60 \%$ had microalbuminuria, which is same as that found by Naser et al. who found that prevalence of MAU was $62.9 \%$ in patients with CAD55. In the PREVEND study (16), 32.8\% IHD patients had microalbuminuria while in HOPE study (17) $20.4 \%$ of patients with cardiovascular disease had microalbuminuria. Of the 30 patients with microalbuminuria, $61.5 \%$ were males and $38.5 \%$ were females. Most patients with MAU were in the age group of 46-60 years which is in accordance with the study by Menno T. Pruijma et al (18), in which most patients were in the age group of 55-64 years, though they found a slight female dominance contrary to our study.

In our study 39 patients were hypertensive, out of which $66.7 \%$ had MAU and $36.4 \%$ of normotensive patients had MAU ( $\mathrm{P}<0.005)$. In a study by Menno T. Pruijma 7\% normotensive and 24\% hypertensive patients had MAU (18). In a study by Michael Bohm et al, 58.4\% hypertensive patients in their study had MAU which is comparable to that seen in our study (19). In our study, out of 50 patients 33 were smokers. Out of this $63.6 \%$ had MAU whereas among non-smokers $52.9 \%$ had MAU. This showed that there was no significant association between smoking and MAU. Similar results were obtained by Menno T. Pruijma et al in their study on general population of Seychelles (18).As for smoking, no significant association was seen between MAU and family history of IHD and previous cardiac events.

\section{Conclusion:-}

From our study we concluded that there is high prevalence of microalbuminuria in non-diabetic ischemic heart disease patients but the association between two is not statistically significant. Our study also showed that there is a strong association between microalbuminuria and hypertension, high LDL cholesterol, and low HDL cholesterol, while it is not significantly associated with smoking, family history of IHD and total cholesterol.

From our study we were not able to find out whether microalbuminuria was independently associated with IHD or its high prevalence was due to the presence of other risk factors of IHD to which microalbuminuria was strongly associated. So from our study we concluded that at present microalbuminuria cannot be used as an independent marker of IHD nor can it be used as a screening tool for IHD in general population unless further prospective studies with large sample size and comparative analysis against normal controls are done in future.

Table 1:-Levels of microalbuminuria (mg/day)

\begin{tabular}{|l|l|l|}
\hline Microalbuminuria & Frequency & $\%$ \\
\hline$<=20$ & 20 & 40 \\
\hline$>20$ & 30 & 60 \\
\hline Total & 50 & 100 \\
\hline
\end{tabular}

Table 2:-Association of microalbuminuria with other risk factors of IHD:

\begin{tabular}{|c|c|c|c|c|c|c|}
\hline & \multirow[b]{2}{*}{ TOTAL } & \multicolumn{2}{|l|}{$\begin{array}{l}\mathrm{MA}<=20 \\
\mathrm{n}=20\end{array}$} & \multicolumn{2}{|l|}{$\begin{array}{l}\mathrm{MA}>20 \\
\mathrm{~N}=30\end{array}$} & \multirow[b]{2}{*}{$\mathrm{p}$ value } \\
\hline & & Frequency & $\%$ & Frequency & $\%$ & \\
\hline H/o of Smoking & 33 & 12 & $36.4 \%$ & 21 & $63.6 \%$ & 0.027 \\
\hline Hypertension & 39 & 13 & $33.3 \%$ & 26 & $66.7 \%$ & 0.003 \\
\hline Family H/O IHD & 15 & 8 & $53.3 \%$ & 7 & $46.7 \%$ & 0.715 \\
\hline $\begin{array}{l}\text { Previous cardiac } \\
\text { event }\end{array}$ & 20 & 8 & $40 \%$ & 12 & $60 \%$ & 0.206 \\
\hline Cholesterol>150 & 25 & 9 & $36 \%$ & 16 & $64 \%$ & 0.048 \\
\hline LDL $>100$ & 19 & 6 & $31.6 \%$ & 13 & $68.4 \%$ & 0.005 \\
\hline Triglycerides $>200$ & 7 & 3 & $42.9 \%$ & 4 & $57.1 \%$ & 0.593 \\
\hline HDL $<35$ & 19 & 4 & $21.1 \%$ & 15 & $78.9 \%$ & 0.004 \\
\hline
\end{tabular}




\section{References:-}

1. Alvin CP. Diabetes Mellitus. In: Dennis LC, Anthony SF, Dan LL, Eugene B, Stephen LH, Jamenson IL, editors. Harrison's principles of Internal Medicine.15th edn: New York: McGraw-Hill; 2001: 2109-2137.

2. Yudkin JS, Forest RD, Caroline AJ. Microalbuminuria as a predictor of vascular disease in nondiabetic subjects. The Lancet 1988; 531-33.

3. Haffner SM, Stein MP, Grubber KK, Hazuda HP, Mitchell BD, Paterson JK. Microalbuminuria: potential marker for increased cardiovascular risk factors in nondiabetic subjects? Arteriosclerosis 1990; 10: 727-31.

4. Gosling P, Hughes EA, Reynolds TM, Fox JP. Microalbuminuria is an early response following myocardial infarction. Eur Heart J. 1991; 12:508-513.

5. Gosling P, Sutcliffe AJ, Cooper MACS, Jones AF. Burn and trauma associated proteinuria:

6. The role of lipid peroxidation and myoglobin. Annals Clin Biochem. 1988; 25: 53-59.

7. Gosling P, Shearman Clifford P. Microproteinuria: response to operation. BMJ, 1988; 296: 338-339.

8. Shearman CP, Gosling P, Walker KJ. Is low level proteinuria a predictor of severity in acute

9. Pancreatitis? J Clin Path. 1989; 42: 1132-5.

10. Mahmud N, Stinson J, O'conell M, Feeley J, Weir GR and Kelleher D, Microalbuminuria is disease activity marker for inflammatory bowel disease. Gut 1993; 34: 5- 24.

11. Gosling P. Microalbuminuria: a sensitive indicator of non-renal disease Ann. Cl. Biochem. 1995; $32: 439$-441.

12. Gosling P. Microalbuminuria and cardio vascular risk - a word of caution. J Hum

13. Gosling P. Microalbuminuria: yet another cardiovascular risk factor. Ann Clin Biochem. 1999; 36: $700-703$.

14. Berton G, Cordiano R, Palmieri R, Cucchini F, Weir GD and Kellcher D. Albumin excretion rate increases during acute myocardial infarction and strongly predicts early mortality.Circulation 1997; 96(10) : 3338-3345.

15. Berton G, Cordiano R, Palmeri R, Cucchni F, De Toni R, Palatini P. Microalbuminuria during acute myocardial infarction; a strong predictor for 1 year mortality. Eur Heart J. 2001; 22(16): 1466-75.

16. Jensen JS. Microalbuminaria and the risk of atherosclerosis.Clinical, epidemiological and physiological investigations. Dan Med Bull 2000; 47(2):63-78

17. Hallan H, Romunstadt S, Kvenild K, and Holman J. Microalbuminuria in diabetic and hypertensive patients and the general population. Scand J Urol Nephrol 2003; 37:151-158.

18. Gerstein HC, Mann JF, Yi Q, Zinman B, Dinneen SF, Hoogwerf B, Halle JP, Young J, Rashkow A, Joyce C, Nawaz S, Yusuf S: Albuminuria and risk of cardiovascular events, death, and heart failure in diabetic and nondiabetic individuals. JAMA 286: 421-426, 2001.

19. Jones CA, Francis ME, Eberhardt MS, Chavers B, Coresh J, Engelgau M, Kusek JW, Byrd

20. Holt D, Narayan KM, Herman WH, Jones CP, Salive M, Agodoa LY: Microalbuminuria in the

21. US population: Third National Health and Nutrition Examination Survey. Am J Kidney Dis 39:445-459, 2002.

22. Menno T. Pruijma, George Madeleineb, Walter F. Riesenc, Michel Burniera and Pascal Bovetb. Prevalence of microalbuminuria in the general population of Seychelles and strong association with diabetes and hypertension independent of renal markers. Journal of Hypertension 2008, 26:871-877.

23. Michael Bohm, Martin Thoenes, Nicolas Danchin, Peter Bramlage, Pablola Puerta and Massimo Volpe. Association of cardiovascular risk factors with microalbuminuria in hypertensive individuals: the i- SEARCH global study. Journal of Hypertension 2007, vol 25: 2317 - 2324.

24. V Nesar Hoseini, O Taziki. Relationship between microalbuminuria and severity of coronary artery disease in non-diabetic patients. ARYA Atherosclerosis Journal 2008, 4(1): 13-16

25. Cummings MH, Watts GF, Tavakolian AF. Prevalence of microalbuminuria, lipoprotein (a) and coronary artery disease in the lipid clinic. J clin Pathol. 1996 Jan; (1): 19-23.

26. Sathisha T.G., Manjunatha Goud B.K., Avinash S.S., Jeevan Shetty, Oinam Sarsina Devi, Devaki R.N. Microalbuminuria in Non-diabetic, Non-hypertensive Myocardial Infarction in South Indian Patients with Relation to Lipid Profile and Cardiac Markers Journal of Clinical and Diagnostic Research. 2011 November (Suppl-1), Vol-5(6): 1158-1160. 